

\title{
L'apprenant en didactique est-il un acteur sociologique comme les autres? Le cas de l'appropriation des TIC et de l'informatique
}

\author{
Cédric Fluckiger
}

\section{- To cite this version:}

Cédric Fluckiger. L'apprenant en didactique est-il un acteur sociologique comme les autres? Le cas de l'appropriation des TIC et de l'informatique. La lettre de l'AIRDF, 2014, 55, pp.13-18. hal-01375367

HAL Id: hal-01375367

https://hal.univ-lille.fr/hal-01375367

Submitted on 3 Oct 2016

HAL is a multi-disciplinary open access archive for the deposit and dissemination of scientific research documents, whether they are published or not. The documents may come from teaching and research institutions in France or abroad, or from public or private research centers.
L'archive ouverte pluridisciplinaire HAL, est destinée au dépôt et à la diffusion de documents scientifiques de niveau recherche, publiés ou non, émanant des établissements d'enseignement et de recherche français ou étrangers, des laboratoires publics ou privés. 


\section{L'apprenant en didactique est-il un acteur sociologique comme les autres ? Le cas de l'appropriation des TIC et de l'informatique}

Référence : Fluckiger Cédric, (2014), L'apprenant en didactique est-il un acteur sociologique comme les autres ? Le cas de l'appropriation des TIC et de l'informatique, La lettre de l'AIRDF, juin 2014, p. 13-18.

\section{Cédric Fluckiger}

\section{Théodile-CIREL (EA4354) Université Lille 3}

Didactique du français et didactique de l'informatique présentent la caractéristique commune de se trouver toutes deux confrontées à la présence importante de pratiques extrascolaires des élèves. Bien que la première ait dû procéder à un travail de légitimation (sociale et scientifique) de ces pratiques (Reuter, 2001), alors que la seconde tend plutôt à relativiser les discours de sens commun sur les "natifs numériques " (Prensky, 2001), toutes deux ont donc dû se saisir de la question des pratiques personnelles, familières aux élèves. Ce faisant, elles ont dû poser la question de la légitimité de leur perspective propre sur cet objet.

Que la didactique soit légitime et armée pour traiter des pratiques personnelles des élèves pourrait en effet ne pas aller de soi et l'on pourrait estimer qu'elles relèvent surtout du domaine de la sociologie. Un regard didactique est pourtant possible, constitue un point de vue particulier sur ces pratiques. En revanche, l'articulation avec un regard sociologique sur ces pratiques n'est pas sans poser problème aux chercheurs. C'est ce que je veux montrer ici en prenant appui sur mon propre parcours de thèse et sur les pistes qu'il m'a conduit à explorer ensuite en didactique. J'en présenterai d'abord quelques éléments afin de préciser en quoi une articulation entre didactique et sociologie m'était apparue nécessaire, en quoi elle a pu s'avérer problématique, avant de généraliser le propos sur la manière dont ces disciplines envisagent le « sujet » didactique et l'« acteur » sociologique.

\section{L'APPROPRIATION DES TIC: QUESTION DIDACTIQUE, REGARD SOCIOLOGIQUE}

Ma thèse de doctorat (Fluckiger, 2007) visait à apporter des éléments de compréhension sur le processus d'appropriation des ordinateurs et des outils informatiques par des élèves de collège, en prêtant attention à la manière dont des compétences spécifiques se forment à travers des usages dans des contextes sociaux variés (la famille, le groupe de pairs et l'école).

Deux ordres de raison m'ont alors conduit à penser que l'articulation sociologie/didactique était particulièrement pertinente pour l'étude des pratiques numériques des adolescents.

En premier lieu, les pratiques numériques extrascolaires, " familières » des enfants et des adolescents, jouent un rôle crucial dans leur construction identitaire, dans leurs sociabilités, dans le bouleversement des hiérarchies culturelles (Lahire, 2002 ; Pasquier, 2005). En conséquence, la manière dont les outils numériques et Internet instrumentent leur rapport aux savoirs, aux autres et à eux-mêmes a des répercussions possibles dans le champ scolaire. Tout enseignant perçoit cette irruption des outils, depuis les SMS envoyés en classe par les élèves (Schneider, 2011) jusqu'aux stratégies complexes de copier-coller de contenus issus d'Internet (Le Douarin \& Delaunay-Teterel, 2012). Ces effets sur les pratiques de classe rendent nécessaire l'examen de ces enjeux, y compris dans les espaces extrascolaires a priori moins familiers au didacticien. 
En outre, bien qu'aucune discipline informatique ne soit présente dans le système éducatif primaire et secondaire français, certains enseignements existent, notamment en technologie ou en documentation. Des usages disciplinaires sont donc répertoriés (de l'expérimentation assistée par ordinateur aux laboratoires de langues), et un mécanisme de certification des compétences a été mis en place entre 2000 et 2006, par l'instauration d'un dispositif : le B2 $\mathrm{i}^{1}$. Mais, en l'absence d'une discipline instituée, les contenus liés à l'informatique à l'école y occupent une place plus incertaine. Ainsi sont-ils construits en référence aux pratiques extrascolaires (comme « Je sais envoyer et recevoir un message "), sans doute davantage que dans les disciplines bien " établies » et plus légitimes dans le champ scolaire. Autre conséquence de cette position incertaine : dans les instructions officielles, leurs finalités mêmes sont systématiquement rattachées non pas aux besoins des apprenants, ni même à ceux des élèves, mais à ceux des « futurs citoyens » (Fluckiger, 2011).

Se posait donc à l'évidence la question didactique de l'organisation de ces apprentissages dans les différentes disciplines, et de leur articulation à la progression des usages et des compétences dans les pratiques personnelles d'élèves qui utilisaient l'ordinateur et Internet essentiellement hors de l'école. Ces préoccupations curriculaires faisaient ressortir la nécessité d'une meilleure compréhension de ce que les élèves faisaient et savaient faire avec les technologies numériques, de ce qu'ils comprenaient des processus informatiques sous-jacents aux applications grand public, des représentations qu'ils construisaient dans leurs usages ludiques et communicationnels familiers.

C'est pourquoi j'ai été amené à effectuer cette thèse conjointement dans un laboratoire de didactique, STEF (ENS de Cachan et INRP) et dans un laboratoire de sociologie des usages (SUSI, France Telecom R\&D).

La sociologie des usages a depuis longtemps fait des phénomènes d'appropriation l'un de ses objets d'étude privilégiés. Issue du champ de la communication, elle vise à étudier les nouveaux objets de communication (Jouet, 2000) en focalisant son attention sur les dynamiques d'usage, la singularité des itinéraires d'appropriation, les écarts entre usages prescrits et usages effectifs, les significations d'usages et l'imaginaire technique, etc. En mettant l'accent sur la dimension socialement construite des usages, cette sociologie cherche à lutter contre les tendances récurrentes au déterminisme technologique, qui pose le problème de l'impact des TIC sur la société, postulant ainsi une certaine autonomie de l'évolution technique, et au déterminisme social qui lui fait pendant (Millerand, 1999). Les mécanismes d'appropriation sont ainsi étudiés dans leur inscription au sein d'un ensemble de relations sociales. Sont mis en avant les effets structurants des ajustements interactionnels entre les membres de la famille (Le Douarin, 2004), ou encore l'importance cruciale jouée par l'évolution des formes de sociabilité juvénile sur les usages des outils de communication numériques (MettonGayon, 2009 ; Pasquier, 2005).

\section{ENJEUX ET DIFFICULTES DE L'ARTICULATION DIDACTIQUE ET SOCIOLOGIE}

Articuler didactique et sociologie me semblait répondre à trois enjeux majeurs : social, méthodologique et théorique, chacun soulevant une série de difficultés.

Il y a un premier enjeu, social, à ne pas subir la traditionnelle " division du travail » (Lahire, 2007) entre sociologues et didacticiens. Lahire (2012) dénonce ainsi les risques d'un découpage

\footnotetext{
${ }^{1}$ Brevet Informatique et Internet, circulaire ${ }^{\circ} 42$ du 7-11-2006.
} 
disciplinaire trop rigide, qui, en refusant de produire des "visions d'ensemble ", laisserait le terrain d'expression à des discours "sans données ni méthode " dus à des idéologues, des essayistes, des journalistes, etc. C'est bien le cas des discours actuels qui construisent à l'envi la figure du « digital native ", invariablement branché, connecté, compétent, bien éloignée des résultats de la recherche (Fluckiger, 2011 ; Cordier, 2011).

Ensuite, il était méthodologiquement délicat d'observer le niveau fin de l'apprentissage d'une notion, d'un savoir-faire, bref, d'un contenu en même temps que le niveau plus macro de l'évolution des usages avec l'âge. Comment en effet observer ce processus d'appropriation des ordinateurs ? Il se déroule dans un temps qui nous était inconnu à l'avance, selon une temporalité variable suivant les individus, dans des lieux variés (en classe, au collège, à domicile) et des contextes sociaux divers ; il passe aussi bien par un effort solitaire que par la mise en place et l'entretien de réseaux de solidarité sociotechnique (Lelong, 2002). J'ai fait le choix d'une démarche ethnographique permettant de suivre les mêmes élèves dans diverses sphères sociales - classe, CDI, centre social, cours de récréation, etc. - pour appréhender différents contextes d'usage possible (apprentissage scolaire, usage ludique individuel, sociabilité numérique, etc.). Mais il s'est avéré que, si les évolutions des usages pouvaient être saisies, les apprentissages demeuraient plus difficiles à appréhender, la familiarité même des outils informatiques rendant difficile la perception par les utilisateurs des mécanismes mis en jeu.

Enfin, la construction du sujet est apparue comme un enjeu théorique fort de la recherche, pour deux raisons distinctes. Tout d'abord, est devenu manifeste au cours de la recherche que l'importance des déterminants sociaux, notamment l'âge et le milieu socioculturel, tendait à masquer d'autres mécanismes à l'œuvre, notamment ceux liés au fonctionnement des contenus informatiques au sein des espaces disciplinaires et transversaux. Plus précisément, la centration sur les trajectoires individuelles reléguait au second plan la manière dont les élèves " vivaient » les contenus scolaires (apprentissage du tableur en technologie, recherches documentaires avec l'enseignant documentaliste, etc.) et leurs organisations, disciplinaires ou transversales. Pour le dire autrement, une centration sur la trajectoire porte le risque de réifier et naturaliser les situations scolaires (disciplines, dispositifs, etc.) que traversent les individus. Ensuite, puisque les enfants et adolescents utilisent les mêmes outils numériques à la fois dans leur sphère familiale, avec leurs pairs et lors d'activités scolaires, il est nécessaire de penser et de construire théoriquement cette pluralité des usages et les éventuels transferts entre eux, qu'ils soient ou non possibles. C'est ce que, inspiré par les travaux de sociologie dispositionnelle de Lahire $(2002,2004)$, j'ai cherché à décrire comme une pluralité intra-individuelle des manières de voir, de faire et d'agir.

\section{OUVERTURE : QUELQUES LEÇONS POUR LES DIDACTIQUES DISCIPLINAIRES \\ Ce retour sur ce parcours de thèse me donne l'occasion de préciser ce qui, à partir de cette expérience singulière, me semble caractériser tout à la fois l'articulation possible mais aussi les limites et les difficultés du croisement des perspectives sociologiques et didactiques.}

Beaucoup a été dit sur le partage du travail entre sociologie et didactique (voir Lahire, 2007) et sur la relative indifférence dans laquelle elles se tiennent mutuellement. On sait les limites d'un tel partage : la didactique ne se réduit pas, en effet, aux questions de transposition des savoirs, pas plus que la sociologie ne se limite à l'étude des structures sociales. Une fois ceci acquis, il est usuel de mettre en avant les différences accordées à « la place du savoir, des formes d'enseignement et des 
processus d'apprentissage au sein de deux disciplines " (Lahire, 2012). C'est cependant une autre dimension que je souhaite discuter ici, à savoir les manières respectives de construire le sujet (sujet didactique, acteur sociologique) dans les deux disciplines.

Construire un objet de recherche, c'est donner le primat à une ou plusieurs dimensions de cet objet : le gaz du chimiste et celui du physicien sont bien le même objet physique, mais ce ne sont pas les mêmes propriétés ni les mêmes relations qui sont mises en avant. Construire le sujet, c'est en effet toujours :

- le contextualiser (dans un ensemble de rapports sociaux, de classes, de pouvoirs, dans des institutions, des espaces disciplinaires, etc.) ;

- le doter de caractéristiques construites théoriquement (dispositions, schèmes, habitus, rapport au savoir, représentations, conscience disciplinaire, etc.) ;

- renvoyer ses conduites observées à un système explicatif (par la culture et les rapports sociaux, par le fonctionnement et l'organisation curriculaire des contenus, etc.).

Il ne s'agit donc pas pour le didacticien de " se défaire du modèle de "I'Élève" en tant qu'être générique, universel, abstrait » (Lahire, 2012) : les sujets didactiques, c'est-à-dire les sujets " en tant qu'ils sont constitués par des relations d'enseignement ou d'apprentissage institutionnalisées, à des objets de savoirs, référés à des disciplines " (Reuter, 2007/2010) ne sont pas plus " abstraits " que les acteurs sociologiques. Ils sont construits différemment ${ }^{2}$.

Ceci m'amène à deux remarques conclusives.

En premier lieu, c'est tout à la fois le choix de ce qui fait contexte, de ce qui est à expliquer et de ce qui explique qui fait le regard didactique ou sociologique. Si le concept d'identité, par exemple, n'a pas connu en didactique l'inflation analytique qu'il a connu en sociologie, c'est sans doute parce qu'une discipline qui se centre sur l'apprentissage est davantage sensible aux « bougés » qu'aux " identités »; et c'est aussi, probablement, parce que c'est un concept qui permet mal de saisir les relations aux autres entités du système didactique, notamment les contenus. Là où le sociologue cherche à expliquer " le social par le social », selon l'expression de Durkheim, les didactiques définissent les sujets par et pour leurs relations aux contenus, dans une approche fondamentalement systémique. Dans sa démarche empirique, le didacticien peut alors privilégier ou non la prise en compte de variables socioculturelles, mais il peut également, sans préjuger d'un système explicatif $a$ priori, laisser au sujet la responsabilité de l'explication de ses conduites (et obtenir des réponses du type « je suis mauvais en français parce que mes parents sont immigrés », " parce que je suis une fille », " parce que le prof n'est pas intéressant », " parce que ça ne sert à rien », etc.).

Ensuite, dans les deux disciplines, l'accent peut être mis sur l'unité ou au contraire l'hétérogénéité des sujets. En attirant l'attention sur la pluralité des conduites dans différents univers sociaux, le recours à la sociologie dispositionnelle $\mathrm{m}^{\prime}$ a donc conduit, par une autre voie, à partager certaines

\footnotetext{
${ }^{2}$ C'est bien la construction des objets de recherche et le projet de connaissance au départ de ces constructions qui différencient le plus nettement ces deux disciplines. La question déborde donc celle de la prise en compte plus ou moins importante du socioculturel dans les didactiques et en sociologie. Il n'est bien sûr pas question, pour le didacticien, de nier que le socioculturel a des effets massifs sur les apprentissages ou que les didactiques (et pas uniquement celle du français) sont «historiquement et structurellement confrontée[s] aux questions liées au socioculturel (Daunay, Delcambre \& Reuter, 2009, p. 20). Mais les didactiques affirment en revanche qu'une trop grande centration sur ces préoccupations ne serait pas compatible avec leur projet de connaissance.
} 
préoccupations des travaux sur les pratiques extrascolaires de la lecture et de l'écriture (Penloup, 2006 ; Reuter, 2001). Considérer ces pratiques comme des objets légitimes en didactique constitue bien un déplacement, en ce sens que cela fait entrer l'enfant, doté d'une épaisseur sociale, et non plus simplement l'élève, dans le système didactique. Ce déplacement ouvre la voie à des recherches sur la manière dont des sujets spécifiques peuvent émerger ou être construits dans différents espaces : élève dans l'espace scolaire (entendu au sens de l'école mais aussi de la forme scolaire), sujet didactique dans l'espace disciplinaire, c'est-à-dire sujet engagé dans certains rapports (d'attirance, de répulsion, d'intérêt, de compréhension...) à des contenus d'enseignement. Le projet ENFELAP (voir notamment Daunay \& Fluckiger, 2011), mais aussi les travaux autour du concept de conscience disciplinaire (Fluckiger et Reuter, 2014 ; Reuter, 2007 ; Cohen-Azria, Lahanier-Reuter \& Reuter, 2013).

\section{Bibliographie}

Cédric FLUCKIGER, Théodile-CIREL (EA 4354)

COHEN-AZRIA, C., LAHANIER-REUTER, D., \& REUTER, Y. (Eds.) (2013). Conscience disciplinaire : les représentations des disciplines à la fin de l'école primaire. Rennes : Presses Universitaires de Rennes.

CORDIER, A. (2011). Pratiques documentaires des élèves et formations dispensées par les enseignants documentalistes : à la croisée des imaginaires d'internet et des pratiques formelles et informelles. (Thèse de doctorat). Université Lille 3, Lille, France.

DAUNAY, B., DELCAMBRE, I., \& REUTER, Y. (dir.) (2009). Didactique du français, le socioculturel en question. Lille : Presses Universitaires du Septentrion.

DAUNAY, B., \& FLUCKIGER, C. (dir.) (2011). Enfant, Élève, Apprenant. Recherches en Didactiques, 11. Lille : Presses Universitaires du Septentrion.

FLUCKIGER, C. (2007). L'appropriation des TIC par les collégiens dans les sphères familières et scolaires. (Thèse de doctorat en Sciences de l'Education.) ENS de Cachan, Cachan, France.

FLUCKIGER, C. (2011). La didactique de l'informatique et les constructions sociales de la figure des jeunes utilisateurs. Recherches en Didactiques, 11, 67-84.

FLUCKIGER, C. \& REUTER, Y. (2014). Les contenus « informatiques » et leur(s) reconstruction(s) par des élèves de CM2. Etude didactique, Recherches en Education, $\mathrm{n}^{\circ} 18,64-78$, http://www.recherches-en-education.net/IMG/pdf/REE-no18.pdf

JOUET, J. (2000). Retour critique sur la sociologie des usages. Réseaux, 18(100), 487-522.

LAHIRE, B. (2002). Portraits sociologiques. Dispositions et variations individuelles. Paris : Nathan.

LAHIRE, B. (2004). La culture des individus. Dissonances culturelles et distinction de soi. Paris : La Découverte.

LAHIRE, B. (2007). La sociologie, la didactique et leurs domaines scientifiques. Éducation et didactique, 1/1, 73-82.

LAHIRE, B. (2012). De la division du travail scientifique : les rapports entre la didactique et la sociologie en période d'hyperspécialisation. Conférence de clôture, "Sociologie et didactiques : vers une transgression des frontières ? », Colloque international Sociologie et Didactiques, Haute Ecole Pédagogique de Vaud Lausanne, 13 \& 14 septembre 2012.

LE DOUARIN, L. (2004). L'ordinateur et les relations père-fils, in D. Le Gall (dir.) Genres de vie et intimités. Chronique d'une autre France II, Paris, L'Harmattan.

LE DOUARIN, L. \& DELAUNAY-TETEREL, H. (2012). Le « net scolaire » à l'épreuve du temps « libre » des lycéens. Revue Française de Socio-Économie, 2011/2, 8, 103-121. 
METTON-GAYON, C. (2009). Les adolescents, leur téléphone et Internet. "Tu viens sur $M S N$ ? » Paris : L'Harmattan.

MILLANRAND, F. (1999). Usages des NTIC, les approches de la diffusion, de l'innovation et de l'appropriation ( $1^{\mathrm{re}}$ et $2^{\mathrm{e}}$ parties). COMMposite, 98/1 et 99/1.

PASQUIER, D. (2005). Cultures lycéennes. La tyrannie de la majorité. Paris : Autrement.

PENLOUP, M.-C. (2006). Pratiques langagières scolaires/non scolaires. La question se pose aussi pour l'écrit. Revue de didactologie des langues-cultures et de lexiculturologie, $141,211-222$.

PRENSKY, M. (2001). Digital Natives, digital immigrants. Do they really think differently. On the Horizon, 9/6.

REUTER, Y. (2001). La «prise en compte» des pratiques extrascolaires de lecture et d'écriture : problèmes et enjeux. Repères, 23, 9-32.

REUTER, Y. (2007). La conscience disciplinaire. Présentation d'un concept. Éducation et didactique, 1/2, 57-71.

REUTER, Y. (dir.) (2010). Dictionnaire des concepts fondamentaux des didactiques. Bruxelles : de Boeck. ( $1^{\text {re }}$ édition 2007)

SCHNEIDER, E. (2011). Travail enseignant, écriture et TIC : comment les économies scripturales gouvernent les pratiques. Colloque international INRP Le travail enseignant au XXI ${ }^{e}$ siècle. Perspectives croisées : didactiques et didactique professionnelle, mars 2011. 